\title{
Arbitrary $l$-Solutions of the Schrodinger Equation in Arbitrary Dimensions for the Energy Dependent Generalized Inverse Quadratic Yukawa Potential
}

\author{
P. O. Ushie, C. M. Ekpo, T. O. Magu, and P. O. Okoi
}

\section{ABSTRACT}

Within the framework of Nikiforov-Uvarov method, we obtained an approximate solution of the Schrodinger equation for the Energy Dependent Generalized inverse quadratic Yukawa potential model. The bound state energy eigenvalues for were computed for various vibrational and rotational quantum numbers. Special cases were considered when the potential parameters were altered, resulting into Energy Dependent Kratzer and Kratzer potential, Energy Dependent Kratzer fues and Kratzer fues potential, Energy Dependent Inverse quadratic Yukawa and Inverse quadratic Yukawa Potential, Energy Dependent Yukawa (screened Coulomb) and Yukawa (screened Coulomb) potential, and Energy Dependent Coulomb and Coulomb potential, respectively. Their energy eigenvalues expressions and numerical computations agreed with the already existing literatures.

Keywords: Energy-dependent potential, Kratzer potential, Nikiforov-Uvarov (NU) method, Yukawa potential.

\section{INTRODUCTION}

Wave equations with energy dependent potentials have been in existence for over 80 years. They can be seen in relativistic quantum mechanics considering particle in an external electromagnetic field [1]-[3]. Energy-dependent potential has been studied in nonrelativistic and relativistic quantum mechanics [4]-[10]. Recently, researchers have showed renewed interest in the study of Energy Dependent Potential (in both relativistic and non-relativistic regime), some of the study amongst others are; [11] studied the Schrödinger equation in D-dimensions for an energydependent Hamiltonian that linearly depends on energy and quadratic on the relative distance using the Nikiforov-Uvarov formalism.[12] showed the influence of the modification of the scalar product, found in the problems of the energydependent potential, on the physical properties of the harmonic oscillator in one dimension. More so, they discussed the effect of this change on the thermodynamic properties of the oscillator. [13] solved the Dirac equation for
Published Online: April 21, 2021

ISSN: $2684-4451$

DOI :10.24018/ejphysics.2021.3.2.63

\section{P. O. Ushie *}

Department of Physics, Cross River University of Technology, Calabar. Nigeria.

(e-mail: patushie98@gmail.com)

\section{M. Ekpo}

Department of Physics, Cross River

University of Technology, Calabar. Nigeria.

e-mail: chrisekpo25@gmail.com)

\section{T. O. Magu}

(a) Department of Pure and Applied Chemistry, University of Calabar, Calabar, Nigeria.

(b) CAS Key Laboratory of Green Printing, Institute of Chemistry, University of Chinese Academy of Sciences, P. R. China.

(e-mail: tommylife4u@ gmail.com)

P. O. Okoi

Department of Physics, University of Calabar, Calabar, Nigeria.

(e-mail: okoipeter7@ gmail.com)

*Corresponding Author the energy-dependent pseudo-harmonic and Mie-type potentials under the pseudospin and spin symmetries using the supersymmetry quantum mechanics. [4] solved the Dirac equation for the energy-dependent Yukawa potential including a tensor interaction term within the framework of the pseudospin and spin symmetry limits with arbitrary spinorbit quantum number using the Nikiforov-Uvarov method. Ikot et al. [14] solved the energy dependent Kratzer potential within the framework of non-relativistic quantum mechanics. [15] generalized Schrodinger equations that include the position-dependent mass was solved for systems featuring energy-dependent potentials. [16] studied the Dirac equation for an energy-dependent potential in the presence of spin and pseudospin symmetries with arbitrary spin-orbit quantum number $\kappa$. [17] also solved the Dirac equation for the energydependent Coulomb (EDC) potential including a Coulomblike tensor (CLT) potential was studied in the presence of spin and pseudospin symmetries with arbitrary spin-orbit quantum number $\mathrm{k}$ by [16]. This solution was achieved within 
the framework of the asymptotic iteration method. It is worthy to state here that to the best of our knowledge, no one can give a solid answer to the question "what is the most useful form for an Energy Dependent Potential (EDP)?" [16].

The generalized inverse quadratic Yukawa potential (GIQYP) is a superposition of the inverse quadratic Yukawa (IQY) and the Yukawa potential. It is asymptotic to a finite value as $r \rightarrow \infty$ and becomes infinite at $r=0$ [18]. This potential has been solved within the framework of the Proper Quantisation Rule [19] and Eigenfunction was obtained via the Formula Method [20].

The Generalized inverse quadratic Yukawa potential model is of the form [18]:

$V(r)=-V_{0}\left(1+\frac{e^{-\alpha r}}{r}\right)^{2}$

It has been noted that differences do not exist between the behavior of the modified Yukawa potential and the inversely quadratic Yukawa potential [21], [22] or the Yukawa potential [23]. Its application to diverse areas of physics has been of great interest concern in recent times [24], [25]. In addition, several quantum mechanical models have been studied extensively both in the relativistic and non-relativistic terrain by several authors [26]-[34].

The Energy Dependent Generalized inverse quadratic Yukawa potential model is of the form:

$V\left(r, E_{n, l}\right)=-\frac{A\left(1+\eta E_{n, l}\right) e^{-2 \alpha r}}{r^{2}}-\frac{B\left(1+\eta E_{n, l}\right) e^{-\alpha r}}{r}-(1+$

$\left.\eta E_{n, l}\right) C$

where $A=C=V_{0}$ and $B=2 V_{0}$.

The Generalized inverse quadratic Yukawa potential reduces to a constant potential when $A=B=0$.

The study of dimensions plays an important role in many areas of physics and the extension of physical problems to higher dimensional space is of great interest. [34] noted that the exact solutions of both the relativistic and nonrelativistic wave equation with certain physical potential in higher dimensions are remarkably important not only in physics and chemistry, but also in pure and applied mathematics.

The organization of the work is as follows: In the next section, we, the review of the NU in Sect. 3, this method is applied method obtain the bound state solutions. In Sect. 4, we obtain numerical results while in the final section. In Sect. 5 we discuss some special cases and in Sect. 6, we give the concluding remark.

\section{REVIEW OF NIKIFOROV-UVAROV METHOD}

The Nikiforov-Uvarov (NU) method is based on solving the hypergeometric-type second-order differential equations by means of the special orthogonal functions [35]. The main equation which is closely associated with the method is given in the following form [36], [37].

$\psi^{\prime \prime}(s)+\frac{\tilde{\tau}(s)}{\sigma(s)} \psi^{\prime}(s)+\frac{\widetilde{\sigma}(s)}{\sigma^{2}(s)} \psi(s)=0$ where $\sigma(s)$ and $\tilde{\sigma}(s)$ are polynomials at most second-degree, $\tilde{\tau}(s)$ is a first-degree polynomial and $\psi(s)$ is a function of the hypergeometric type.

The exact solution of (2) can be obtained by using the transformation:

$\psi(s)=\phi(s) y(s)$

This transformation reduces Eq. (2) into a hypergeometrictype equation of the form:

$\sigma(s) y^{\prime \prime}(s)+\tau(s) y^{\prime}(s)+\lambda y(s)=0$

The function $\phi(s)$ can be defined as the logarithm derivative:

$\frac{\phi^{\prime}(s)}{\phi(s)}=\frac{\pi(s)}{\sigma(s)}$

where

$\pi(s)=\frac{1}{2}[\tau(s)-\tilde{\tau}(s)]$

with $\pi(s)$ being at most a first-degree polynomial. The second $\psi(s)$ being $y_{n}(n)$ in Eq. (3), is the hypergeometric function with its polynomial solution given by Rodrigues relation:

$y^{(n)}(s)=\frac{B_{n}}{\rho(s)} \frac{d^{n}}{d s^{n}}\left[\sigma^{n} \rho(s)\right]$

Here, $B_{n}$ is the normalization constant and $\rho(s)$ is the weight function which must satisfy the condition:

$(\sigma(s) \rho(s))^{\prime}=\sigma(s) \tau(s)$

$\tau(s)=\tilde{\tau}(s)+2 \pi(s)$

It should be noted that the derivative of $\tau(s)$ with respect to $s$ should be negative. The eigenfunctions and eigenvalues can be obtained using the definition of the following function $\pi(s)$ and parameter $\lambda$, respectively:

$\pi(s)=\frac{\sigma^{\prime}(s)-\tilde{\tau}(s)}{2} \pm \sqrt{\left(\frac{\sigma^{\prime}(s)-\tilde{\tau}(s)}{2}\right)^{2}-\tilde{\sigma}(s)+k \sigma(s)}$

where

$k=\lambda-\pi^{\prime}(s)$

The value of $k$ can be obtained by setting the discriminant of the square root in (9) equal to zero. As such, the new eigenvalue equation can be given as:

$\lambda_{n}=-n \tau^{\prime}(s)-\frac{n(n-1)}{2} \sigma^{\prime \prime}(s), n=0,1,2, \ldots$ 


\section{BOUND STATE SOLUTION WITH ENERGY DEPENDENT GENERALIZED INVERSE QUADRATIC YUKAWA POTENTIAL IN D DIMENSION}

The radial Schrodinger equation in $D$ dimension can be written as [38], [39]:

$\left[\frac{d^{2} R_{n l}}{d r^{2}}-\frac{2 \mu V(r)}{\hbar^{2}}-\frac{(D+2 \ell-1)(D+2 \ell-3)}{4 r^{2}}+\frac{2 \mu E_{n l}}{\hbar^{2}}\right] R_{n l}(r)=0$

where $\mu$ is the reduced mass, $E_{n l}$ is the energy spectrum, $\hbar$ is the reduced Planck's constant and $n$ and $l$ are the radial and orbital angular momentum quantum numbers respectively (or vibration-rotation quantum number in quantum chemistry). Substituting equation (1) into equation (12) gives:

$\left[\frac{d^{2} R_{n l}}{d r^{2}}-\frac{2 \mu}{\hbar^{2}}\left(-V_{0}\left(1+\frac{e^{-\alpha r}}{r}\right)^{2}\left(1+\eta E_{n, l}\right)\right)-\right.$

$\left.\frac{(D+2 \ell-1)(D+2 \ell-3)}{4 r^{2}}+\frac{2 \mu E_{n l}}{\hbar^{2}}\right] R_{n l}(r)=0$

Simplifying further (13) becomes:

$\left[\frac{d^{2}}{d r^{2}}-\frac{2 \mu}{\hbar^{2}}\left(-\frac{A\left(1+\eta E_{n, l}\right) e^{-2 \alpha r}}{r^{2}}-\frac{B\left(1+\eta E_{n, l}\right) e^{-\alpha r}}{r}-(1+\right.\right.$
$\left.\left.\left.\eta E_{n, l}\right) C\right)-\frac{(D+2 \ell-1)(D+2 \ell-3)}{4 r^{2}}+\frac{2 \mu E_{n l}}{\hbar^{2}}\right] R_{n l}=0$

Employing the Pekeris type approximation scheme [40], which is given by:

$\frac{1}{r^{2}}=\frac{4 \alpha^{2} e^{-2 \alpha r}}{\left(1-q e^{-2 \alpha r}\right)^{2}}$ and $\frac{1}{r}=\frac{2 \alpha e^{-\alpha r}}{\left(1-q e^{-2 \alpha r}\right)}$

Equation (14) becomes:

$\frac{d^{2} R_{n \ell}(r)}{d r^{2}}+\frac{1}{\left(1-e^{-2 \alpha r}\right)^{2}}\left[\frac{2 \mu\left(E_{n l}+C\left(1+\eta E_{n, l}\right)\right)}{\hbar^{2}}\left(1-e^{-2 \alpha r}\right)^{2}+\right.$

$\frac{4 \mu B \alpha\left(1+\eta E_{n, l}\right) e^{-2 \alpha r}}{\hbar^{2}}\left(1-e^{-2 \alpha r}\right)+\frac{8 \mu A \alpha^{2}\left(1+\eta E_{n, l}\right) e^{-4 \alpha r}}{\hbar^{2}}-$

$\left.\frac{(D+2 \ell-1)(D+2 \ell-3) 4 \alpha^{2} e^{-2 \alpha r}}{4}\right] R_{n \ell}(r)$

Equation (16) can be simplified into the form and introducing the following dimensionless abbreviations:

$$
\left\{\begin{array}{c}
-\varepsilon_{n}=\frac{\mu\left(E_{n l}+C\left(1+\eta E_{n, l}\right)\right)}{2 \hbar^{2} \alpha^{2}} \\
\beta=\frac{2 \mu A\left(1+\eta E_{n, l}\right)}{\hbar^{2}} \\
\chi=\frac{\mu B\left(1+\eta E_{n, l}\right)}{\hbar^{2} \alpha} \\
\gamma=\frac{(D+2 \ell-1)(D+2 \ell-3)}{4}
\end{array}\right\}
$$

And using the transformation $s=e^{-2 \alpha r}$ so as to enable us to apply the NU method as a solution of the hypergeometric type:

$$
\frac{d^{2} R_{n \ell}(r)}{d r^{2}}=4 \alpha^{2} s^{2} \frac{d^{2} R_{n \ell}(s)}{d s^{2}}+4 \alpha^{2} s \frac{d R_{n \ell}(s)}{d s}
$$

$$
\begin{aligned}
& \frac{d^{2} R_{n \ell}(s)}{d s^{2}}+\frac{1-q s}{s(1-q s)} \frac{d R_{n \ell}(s)}{d s}+\frac{1}{s^{2}(1-q s)^{2}}\left[-s^{2}\left(\varepsilon_{n}-\beta+\chi\right)+\right. \\
& \left.s\left(2 \varepsilon_{n}+\chi-\gamma\right)-\varepsilon_{n}\right] R_{n \ell}(s)=0
\end{aligned}
$$

Comparing (19) and (2), we have the following parameters:

$$
\left\{\begin{array}{c}
\tilde{\tau}(s)=1-s \\
\sigma(s)=s(1-s) \\
\tilde{\sigma}(s)=-s^{2}\left(\varepsilon_{n}-\beta+\chi\right)+s\left(2 \varepsilon_{n}+\chi-\gamma\right)-\varepsilon_{n}
\end{array}\right\}
$$

Substituting these polynomials into (9), we get $\pi(s)$ to be:

$\pi(s)=-\frac{q s}{2} \pm \sqrt{(a-k) s^{2}+(b+k) s+c}$

where

$\left\{\begin{array}{c}a=\frac{1}{4}+\varepsilon_{n}-\beta+\chi \\ b=-\left(2 \varepsilon_{n}+\chi-\gamma\right) \\ c=\varepsilon_{n}\end{array}\right\}$

To find the constant $k$, the discriminant of the expression under the square root of (21) must be equal to zero. As such, we have that:

$k \pm=-(\chi-\gamma) \pm 2 \sqrt{\varepsilon_{n}\left(\frac{1}{4}-\beta+\gamma\right)}$

Substituting Eq. (23) into Eq. (21) yields:

$\pi(s)=-\frac{s}{2} \pm\left[\left(\sqrt{\varepsilon_{n}}+\sqrt{\left(\frac{1}{4}-\beta+\gamma\right)}\right) s-\sqrt{\varepsilon_{n}}\right]$

From the knowledge of NU method, we choose the expression $\pi(s)$ _ which the function $\tau(s)$ has a negative derivative. This is given by:

$k_{-}=-(\chi-\gamma)-2 \sqrt{\varepsilon_{n}\left(\frac{1}{4}-\beta+\gamma\right)}$

with $\tau(s)$ being obtained as:

$\tau(s)=1-2 s-2\left[\left(\sqrt{\left(\frac{1}{4}-\beta+\gamma\right)}+\sqrt{\varepsilon_{n}}\right) s-\sqrt{\varepsilon_{n}}\right](26)$

Referring to (10), we define the constant $\lambda$ as:

$\lambda=-\frac{1}{2}-\left(\sqrt{\left(\frac{1}{4}-\beta+\gamma\right)}+\sqrt{\varepsilon_{n}}\right)+(\gamma-\chi)-$

$2 \sqrt{\varepsilon_{n}\left(\frac{1}{4}-\beta+\gamma\right)}$

Substituting (27) into (11) and carrying out simple algebra, where: 
$\tau^{\prime}(s)=-2\left(1+\left(\sqrt{\left(\frac{1}{4}-\beta+\gamma\right)}+\sqrt{\varepsilon_{n}}\right)\right)<0$

and

$\sigma^{\prime \prime}(s)=-2$

We have

$\varepsilon_{n}=\frac{1}{4}\left[\frac{\left(n+\frac{1}{2}+\sqrt{\frac{1}{4}-\beta+\gamma}\right)^{2}+\beta-\chi}{\left(\left(n+\frac{1}{2}+\sqrt{\frac{1}{4}-\beta+\gamma}\right)\right)}\right]^{2}$

Substituting (17) into (30) yields the energy eigenvalue equation of the Energy Dependent Generalized Inverse Quadratic Yukawa Potential in $D$ dimension in the form:

$E_{n \ell}=-C\left(1+\eta E_{n, l}\right)-$

$\frac{\hbar^{2} \alpha^{2}}{2 \mu}\left[\frac{\left(n+\frac{1}{2}+\sqrt{\frac{1}{4}-\frac{2 \mu A\left(1+\eta E_{n, l}\right)}{\hbar^{2}}+\frac{(D+2 \ell-1)(D+2 \ell-3)}{4}}\right)^{2}+\frac{2 \mu A\left(1+\eta E_{n, l}\right)}{\hbar^{2}}-\frac{\mu B\left(1+\eta E_{n, l}\right)}{\hbar^{2} \alpha}}{\left(n+\frac{1}{2}+\sqrt{\frac{1}{4}-\frac{2 \mu A\left(1+\eta E_{n, l}\right)}{\hbar^{2}}+\frac{(D+2 \ell-1)(D+2 \ell-3)}{4}}\right)}\right]^{2}$

The corresponding wave functions can be evaluated by substituting $\pi(s)_{-}$and $\sigma(s)$ from (24) and (20), respectively into (5) and solving the first order differential equation. This gives:

$\Phi(s)=s^{\sqrt{\varepsilon_{n}}}(1-s)^{\frac{1}{2}+\sqrt{\frac{1}{4}-\beta+\gamma}}$

The weight function $\rho(s)$ from (7) can be obtained as:

$\rho(s)=s^{2 \sqrt{\varepsilon_{n}}}(1-s)^{2 \sqrt{\frac{1}{4}-\beta+\gamma}}$

From the Rodrigues relation of (6), we obtain:

$y_{n}(s) \equiv N_{n, l} P_{n}^{\left(2 \sqrt{\varepsilon_{n}}, 2 \sqrt{\frac{1}{4}-\beta+\gamma}\right)}(1-2 s)$

where $P_{n}^{(\theta, \vartheta)}$ is the Jacobi Polynomial.

Substituting $\Phi(s)$ and $y_{n}(s)$ from (32) and respectively into (3), we obtain:

$\psi_{n}(s)=N_{n, l} s^{\sqrt{\varepsilon_{n}}}(1-s)^{\frac{1}{2}+\sqrt{\frac{1}{4}-\beta+\gamma}} P_{n}^{\left(2 \sqrt{\varepsilon_{n}}, 2 \sqrt{\frac{1}{4}-\beta+\gamma}\right)}(1-$

2s)

\section{Special Cases (Deductions From (31))}

In this section, we take some adjustments of constants in Eq. (1a and $b$ ) to have the following cases:

\section{A. Kratzer Potential}

If $\alpha \rightarrow 0$ and if set $A=-V_{1}, B=2 V_{1}$ and $C=$ $-V_{1}$ Equation $1 \mathrm{c}$ reduces to:

$V(r)=\frac{A\left(1+\eta E_{n, l}\right)}{r^{2}}-\frac{B\left(1+\eta E_{n, l}\right)}{r}+C\left(1+\eta E_{n, l}\right)$

Equation (31) becomes the energy dependent Kratzer Potential in $D$ dimesnsions:

$E_{n \ell}=C\left(1+\eta E_{n, l}\right)-$

$\frac{\mu B^{2}\left(1+\eta E_{n, l}\right)^{2}}{2 \hbar^{2}\left(n+\frac{1}{2}+\sqrt{\frac{1}{4}+\frac{2 \mu A\left(1+\eta E_{n, l}\right)}{\hbar^{2}}+\frac{(D+2 \ell-1)(D+2 \ell-3)}{4}}\right)^{2}}$

If we set $\eta=0$, the energy equation reduces to energy equation for Kratzer potential in $D$ dimesnsions:

$E_{n \ell}=C-\frac{\mu B^{2}}{2 \hbar^{2}\left(n+\frac{1}{2}+\sqrt{\frac{1}{4}+\frac{2 \mu A}{\hbar^{2}}+\frac{(D+2 \ell-1)(D+2 \ell-3)}{4}}\right)^{2}}$

Comments: (38) is the Energy eigenvalue equation for the Kratzer potential in $D$ dimensions. If $D=3$ reduces to energy equation for Kratzer potential in $3 \mathrm{D}$, (45) is very consistent with the result obtained in (125) of [37].

\section{B. Inversely Quadratic Yukawa Potential}

If $B=C=0$ the potential (Equation 1a) reduces to the Inverse Quadratic Yukawa Potential [41].

$V(r)=-\frac{A\left(1+\eta E_{n, l}\right) e^{-2 \alpha r}}{r^{2}}$

$E_{n \ell}=$

$-\frac{\hbar^{2} \alpha^{2}}{2 \mu}\left[\frac{\left(n+\frac{1}{2}+\sqrt{\frac{1}{4}-\frac{2 \mu A\left(1+\eta E_{n, l}\right)}{\hbar^{2}}+\frac{(D+2 \ell-1)(D+2 \ell-3)}{4}}\right)^{2}+\frac{2 \mu A\left(1+\eta E_{n, l}\right)}{\hbar^{2}}}{\left(n+\frac{1}{2}+\sqrt{\frac{1}{4}-\frac{2 \mu A\left(1+\eta E_{n, l}\right)}{\hbar^{2}}+\frac{(D+2 \ell-1)(D+2 \ell-3)}{4}}\right)}\right]^{2}$

If we set $\eta=0$, the energy equation reduces to energy equation for Inverse Quadratic Yukawa Potential in $D$ dimesnsions:

$E_{n \ell}=-\frac{\hbar^{2} \alpha^{2}}{2 \mu}\left[\frac{\left(n+\frac{1}{2}+\sqrt{\frac{1}{4}-\frac{2 \mu A}{\hbar^{2}}+\frac{(D+2 \ell-1)(D+2 \ell-3)}{4}}\right)^{2}+\frac{2 \mu A}{\hbar^{2}}}{\left(n+\frac{1}{2}+\sqrt{\frac{1}{4}-\frac{2 \mu A}{\hbar^{2}}+\frac{(D+2 \ell-1)(D+2 \ell-3)}{4}}\right)}\right]^{2}$

Comments: Equation (41) is the energy equation for the Inverse Quadratic Yukawa Potential in $D$ Dimensions. If $D=$ 3 , (41) reduces to the energy equation in $3 \mathrm{D}$, which is 
identical to the results in; (37) of [42], (18) of [43] and (47) of [44].

\section{Yukawa Potential}

If and $A=C=0$ the potential (Equation 1c) reduces to the Yukawa Potential [4]. [4] solved the Dirac equation for the energy-dependent Yukawa potential including a tensor interaction term within the framework of the pseudospin and spin symmetry limits with arbitrary spin-orbit quantum number. The limiting cases of the model was reduced to the energy-dependent Yukawa and Coulomb potentials, respectively.

$V(r)=-\frac{B\left(1+\eta E_{n, l}\right) e^{-\alpha r}}{r}$

$E_{n \ell}=-\frac{\hbar^{2} \alpha^{2}}{2 \mu}\left[\frac{\left(n+\frac{1}{2}+\sqrt{\frac{1}{4}+\frac{(D+2 \ell-1)(D+2 \ell-3)}{4}}\right)^{2}-\frac{\mu B\left(1+\eta E_{n, l}\right)}{\hbar^{2} \alpha}}{\left(n+\frac{1}{2}+\sqrt{\frac{1}{4}+\frac{(D+2 \ell-1)(D+2 \ell-3)}{4}}\right)}\right]^{2}$

If we set $\eta=0$, the energy equation reduces to energy equation for Yukawa Potential in $D$ Dimensions:

$E_{n \ell}=-\frac{\hbar^{2} \alpha^{2}}{2 \mu}\left[\frac{\left(n+\frac{1}{2}+\sqrt{\frac{1}{4}+\frac{(D+2 \ell-1)(D+2 \ell-3)}{4}}\right)^{2}-\frac{\mu B}{\hbar^{2} \alpha}}{\left(n+\frac{1}{2}+\sqrt{\frac{1}{4}+\frac{(D+2 \ell-1)(D+2 \ell-3)}{4}}\right)}\right]^{2}$

Comments: (44) is the energy eigenvalue equation for the Yukawa potential in $D$ dimensions. If $D=3$, (44) becomes identical with (87) and (15) reported in [45] and [46], respectively.

\section{Kratzer-Feus Potential}

If we set $C=0, \alpha \rightarrow 0,(1 \mathrm{c})$ reduces to the Coulomb plus Inverse-Square Potential [47], [48]:

$V(r)=-\frac{B}{r}+\frac{A}{r^{2}}$

$A=C=-V_{1}$ and $B=2 V_{1}$

$E_{n \ell}=-\frac{2 \mu B^{2}\left(1+\eta E_{n, l}\right)^{2}}{\hbar^{2}\left(2 n+1+\sqrt{\frac{8 \mu A\left(1+\eta E_{n, l}\right)}{\hbar^{2}}+(D+2 \ell-1)^{2}}\right)}$

If we set $\eta=0$, the energy equation reduces to energy equation for Kratzer-Fues Potential in $D$ dimesnsions:

$E_{n \ell}=\frac{-2 \mu B^{2}}{\hbar^{2}\left(2 n+1+\sqrt{\frac{8 \mu A}{\hbar^{2}}+(D+2 \ell-2)^{2}}\right)^{2}}$

Comments: (47) is also known as the Kratzer-Feus potential, this potential was studied by [48] in arbitrary dimensions. If we set $\hbar=\mu=1$, (47) fully agrees with the result reported in eq. (28) of [48]. (37) is also consistent with the result obtained in (15) of [47].

\section{E. Coulomb Potential}

If $A=C=0, \alpha \rightarrow 0$ the potential (Equation 1c) reduces to the Coulomb Potential [34].

$V(r)=-\frac{B}{r}$

$E_{n \ell}=-\frac{\mu B^{2}\left(1+\eta E_{n, l}\right)^{2}}{2 \hbar^{2}\left(n+\frac{1}{2}+\sqrt{\frac{1}{4}+\frac{(D+2 \ell-1)(D+2 \ell-3)}{4}}\right)^{2}}$

If we set $\eta=0$, the energy equation reduces to energy equation for Coulomb Potential in $D$ dimesnsions:

$E_{n \ell}=-\frac{\mu B^{2}}{2 \hbar^{2}\left(n+\frac{1}{2}+\sqrt{\frac{1}{4}+\frac{(D+2 \ell-1)(D+2 \ell-3)}{4}}\right)^{2}}$

Comments: (50) is the energy equation for Coulomb potential in $D$ Dimensions. This result is consistent with the result obtained in (7.14) of [34].

TABLE I: THE BOUND STATE ENERGY LEVELS (IN UNITS OF FM-1) OF THE GIQYP FOR VARIOUS VALUES OF $n, l$ AND FOR $\hbar=\mu=1$,

\begin{tabular}{ccccc}
\multicolumn{5}{c}{$V_{0}=0.5$ and $\alpha=0.001$} \\
\hline$n$ & $l$ & $E_{n l}$ & $E_{n l}$ & $E_{n l}$ \\
\hline 0 & 1 & -0.68960354 & -0.540881135 & -0.506890535 \\
1 & 1 & -0.571807619 & -0.478344718 & -0.45428128 \\
2 & 1 & -0.537127791 & -0.45790939 & -0.436746066 \\
3 & 1 & -0.522410094 & -0.44889584 & -0.428947535 \\
\hline 0 & 2 & -0.563050854 & -0.474126328 & -0.450862611 \\
1 & 2 & -0.53372401 & -0.456181624 & -0.435330535 \\
2 & 2 & -0.520749321 & -0.448032007 & -0.42823602 \\
3 & 2 & -0.513895979 & -0.443670831 & -0.424428249 \\
\hline 0 & 3 & -0.532601875 & -0.455583817 & -0.434834803 \\
1 & 3 & -0.520190696 & -0.447728815 & -0.427983558 \\
2 & 3 & -0.513578774 & -0.4434969 & -0.424283086 \\
3 & 3 & -0.509646332 & -0.440963924 & -0.422065022 \\
\hline 0 & 4 & -0.519902567 & -0.44757064 & -0.427851451 \\
1 & 4 & -0.513414329 & -0.443405797 & -0.424206841 \\
2 & 4 & -0.509543853 & -0.440906841 & -0.422017192 \\
3 & 4 & -0.507053026 & -0.439293102 & -0.42060214 \\
\hline
\end{tabular}

TABLE II: THE BOUND STATE ENERGY LEVELS (IN UNITS OF FM-1) OF THE GIQYP FOR VARIOUS VALUES OF $n, l$ AND FOR $\hbar=\mu=1$,

\begin{tabular}{ccccc}
\multicolumn{5}{c}{$V_{0}=0.5$ and $\alpha=0.01$} \\
$n$ & $l$ & $E_{n l}$ & $E_{n l}$ & $E_{n l}$ \\
& & $\eta=0$ & $\eta=0.3$ & $\eta=0.4$ \\
\hline 0 & 1 & -0.677413346 & -0.533276163 & -0.500165487 \\
1 & 1 & -0.561940037 & -0.471450998 & -0.448062163 \\
2 & 1 & -0.528190997 & -0.451485229 & -0.430921372 \\
3 & 1 & -0.514145048 & -0.442922673 & -0.423531854 \\
\hline 0 & 2 & -0.55338674 & -0.467294042 & -0.444686873 \\
1 & 2 & -0.524911823 & -0.449816583 & -0.429554613 \\
2 & 2 & -0.512594774 & -0.442124652 & -0.422877789 \\
3 & 2 & -0.506388292 & -0.438268817 & -0.419543693 \\
\hline 0 & 3 & -0.523833592 & -0.449241354 & -0.42907784 \\
1 & 3 & -0.512076165 & -0.441846493 & -0.422647408 \\
2 & 3 & -0.506112992 & -0.43812357 & -0.419424336 \\
3 & 3 & -0.502880256 & -0.43616801 & -0.417753812 \\
\hline 0 & 4 & -0.511809303 & -0.441701803 & -0.42252723 \\
1 & 4 & -0.505970901 & -0.438047907 & -0.41936201 \\
2 & 4 & -0.502802325 & -0.43612858 & -0.41772203 \\
3 & 4 & -0.501087669 & -0.435171326 & -0.416932685 \\
\hline
\end{tabular}


TABLE III: THE BOUND STATE ENERGY LEVELS (IN UNITS OF FM-1) OF THE GIQYP FOR VARIOUS VALUES OF $n, l$ AND FOR $\hbar=\mu=1$,

\begin{tabular}{ccccc}
\multicolumn{5}{c}{$V_{0}=1$ and $\alpha=0.001$} \\
\hline$n$ & $l$ & $E_{n l}$ & $E_{n l}$ & $E_{n l}$ \\
& & $\eta=0$ & $\eta=0.3$ & $\eta=0.4$ \\
\hline 0 & 1 & -2.9940045 & -1.095985476 & -0.953730354 \\
1 & 1 & -1.4970045 & -0.909932541 & -0.820368029 \\
2 & 1 & -1.2197845 & -0.843534551 & -0.771214559 \\
3 & 1 & -1.122760125 & -0.814219641 & -0.749065228 \\
\hline 0 & 2 & -1.302201872 & -0.883072695 & -0.80268238 \\
1 & 2 & -1.155363939 & -0.831952923 & -0.763421879 \\
2 & 2 & -1.093938232 & -0.808367119 & -0.745076568 \\
3 & 2 & -1.062548532 & -0.795710385 & -0.735161319 \\
\hline 0 & 3 & -1.143685817 & -0.828799823 & -0.761180195 \\
1 & 3 & -1.088310737 & -0.806742392 & -0.743910796 \\
2 & 3 & -1.059418944 & -0.794772607 & -0.73448474 \\
3 & 3 & -1.042468019 & -0.787587976 & -0.728806789 \\
\hline 0 & 4 & -1.085664968 & -0.805938767 & -0.743328245 \\
1 & 4 & -1.057929862 & -0.794305782 & -0.734144741 \\
2 & 4 & -1.041548733 & -0.787294122 & -0.728592128 \\
3 & 4 & -1.031076397 & -0.782753693 & -0.724989228 \\
\hline
\end{tabular}

TABLE IV: THE BOUND STATE ENERGY LEVELS (IN UNITS OF FM-1) OF THE GIQYP FOR VARIOUS VALUES OF $n, l$ AND FOR $\hbar=\mu=1$,

\begin{tabular}{ccccc}
\multicolumn{5}{c}{$V_{0}=1$ and $\alpha=0.01$} \\
$n$ & $l$ & $E_{n l}$ & $E_{n l}$ & $E_{n l}$ \\
\hline 0 & 1 & -2.940450000 & -1.085469075 & -0.945254024 \\
1 & 1 & -1.470450000 & -0.899255077 & -0.811503393 \\
2 & 1 & -1.198450000 & -0.833117822 & -0.762452911 \\
3 & 1 & -1.103512500 & -0.804156172 & -0.740561286 \\
\hline 0 & 2 & -1.279268339 & -0.872291081 & -0.793693742 \\
1 & 2 & -1.135367365 & -0.821569833 & -0.754665745 \\
2 & 2 & -1.075445609 & -0.798394601 & -0.73663523 \\
3 & 2 & -1.045120125 & -0.786188347 & -0.727102695 \\
\hline 0 & 3 & -1.123949057 & -0.818442092 & -0.752437387 \\
1 & 3 & -1.069983263 & -0.796805959 & -0.735495805 \\
2 & 3 & -1.042124745 & -0.785295278 & -0.726461699 \\
3 & 3 & -1.026091933 & -0.778625634 & -0.721241813 \\
\hline 0 & 4 & -1.067417989 & -0.796021807 & -0.734927697 \\
1 & 4 & -1.040702385 & -0.784852132 & -0.726140704 \\
2 & 4 & -1.025236123 & -0.778359523 & -0.721050146 \\
3 & 4 & -1.015671687 & -0.774402898 & -0.717976939 \\
\hline
\end{tabular}

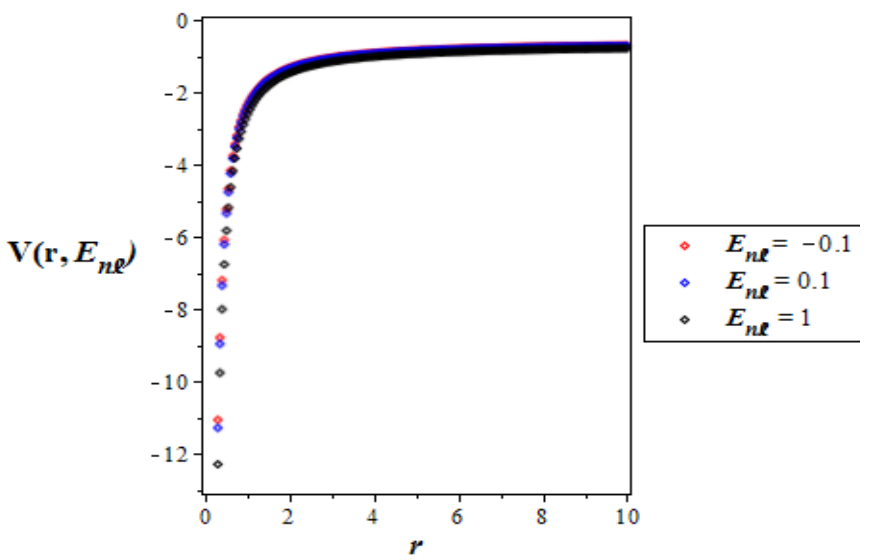

Fig. 1. The variation of the Potential energy for various values of the energy $\left(E_{n l}\right)$ as a function of $r$. We choose $V_{0}=0.5, \eta=0.3$ and

$$
\alpha=0.001 \text {. }
$$

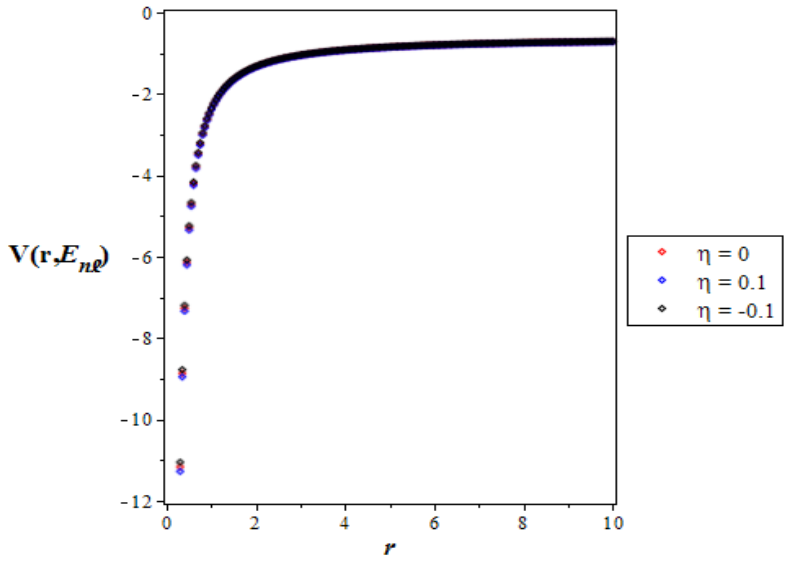

Fig. 2. The variation of the Potential energy for various values of the energy slope parameter $(\eta)$ as a function $(r)$. We choose $V_{0}=0.5$, $E_{n l}=0.1$ and $\alpha=0.001$.

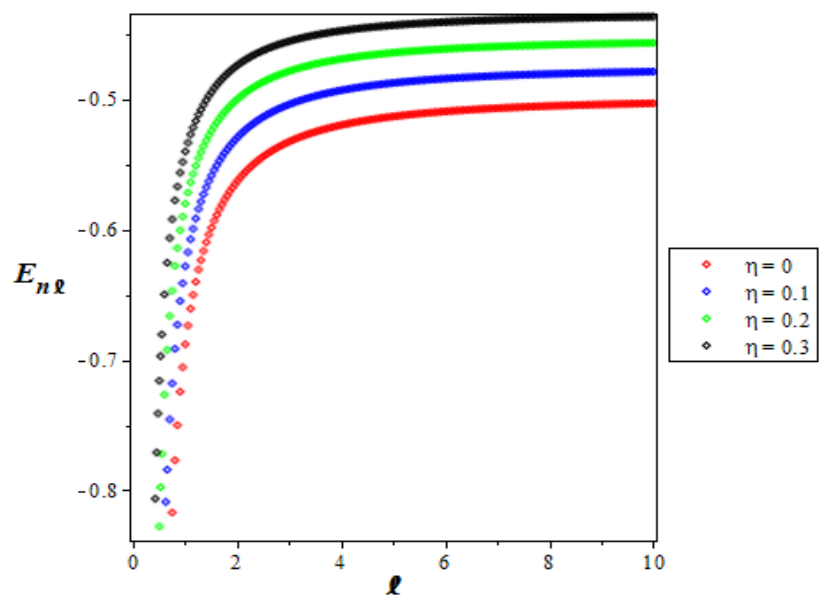

Fig. 3. The variation of the ground state $(n=0)$ energy level for various values of the energy slope $\operatorname{parameter}(\eta)$ as a function of $l$. We choose $V_{0}=0.5$, and $\alpha=0.001$ in $3 D$.

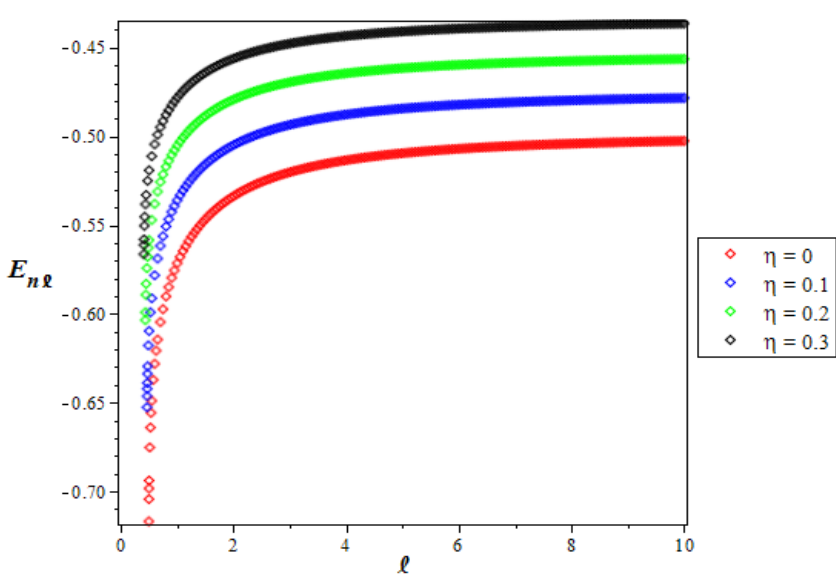

Fig. 4. The variation of the first excited state $(n=1)$ energy level for various values of the energy slope parameter $(\eta)$ as a function of $l$. We choose $V_{0}=0.5$, and $\alpha=0.001$ in $3 D$. 


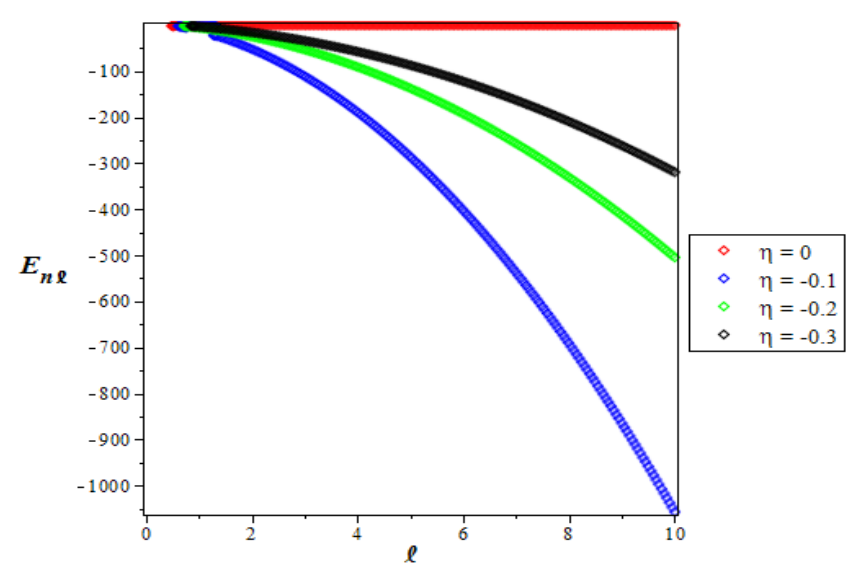

Figure 5: The variation of the ground state $(n=0)$ energy level for various values of the energy slope $\operatorname{parameter}(\eta \leq 0)$ as a function of $l$. We choose $V_{0}=0.5$, and $\alpha=0.001$ in $3 D$.

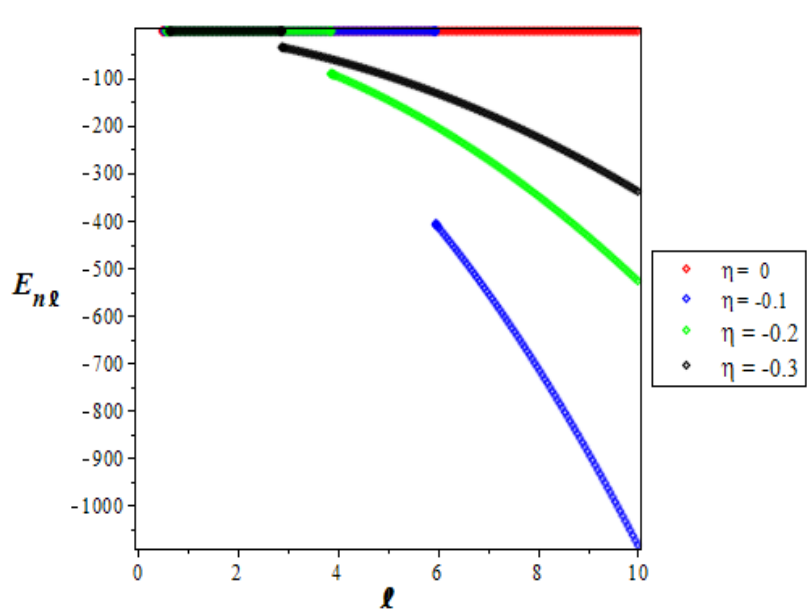

Fig. 6. The variation of the first excited state $(n=1)$ energy level for various values of the energy slope $\operatorname{parameter}(\eta \leq 0)$ as a function of $l$. We choose $V_{0}=0.5$, and $\alpha=0.001$ in $3 D$.

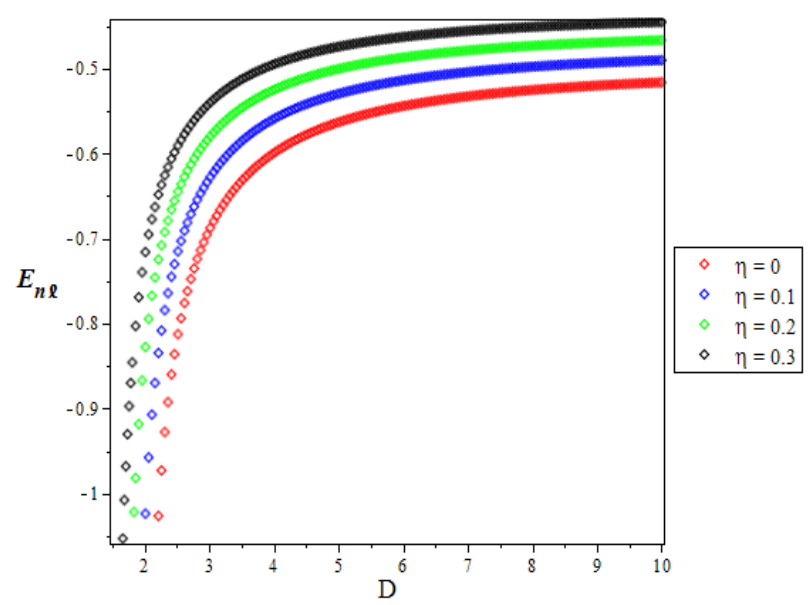

Fig. 7. The variation of the ground state $(n=0)$ energy level for values of the energy slope $\operatorname{parameter}(\eta)$ as a function of $D$. We choose $l=1$, $V_{0}=0.5$, and $=0.001$.

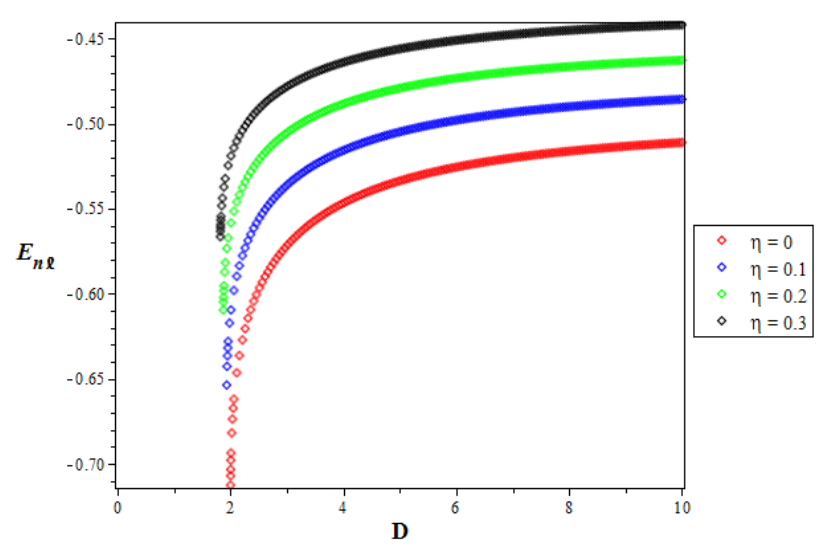

Fig. 8. The variation of the First excited state $(n=1)$ energy level for values of the energy slope $\operatorname{parameter}(\eta)$ as a function of $D$ We choose $l=1, V_{0}=0.5$, and $\alpha=0.001$

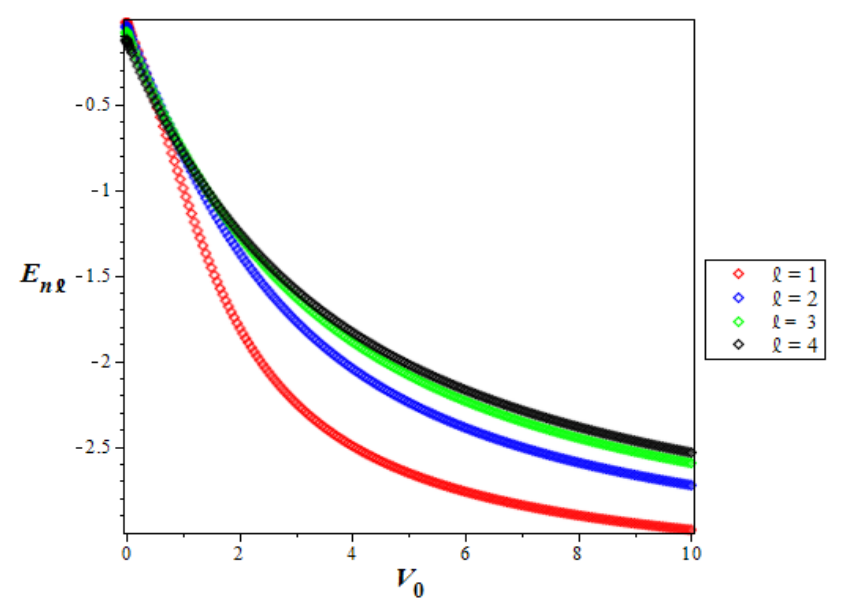

Fig. 9. The variation of the ground state $(n=0)$ energy level for various values of the Rotational Quantum Number $(l)$ as a function of $V_{0}$. We choose $=0.3$, and $\alpha=0.001$ in $3 D$.

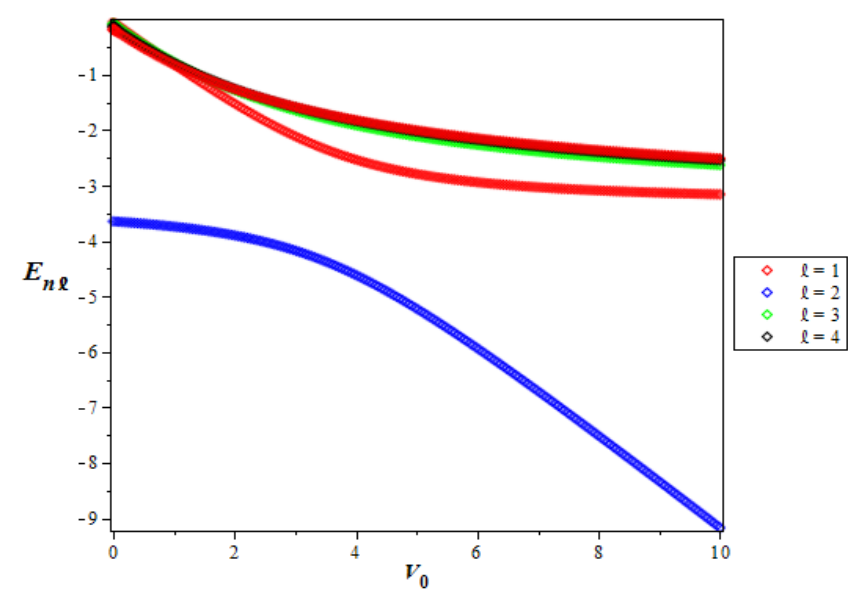

Fig. 10. The variation of the First excited state $(n=1)$ energy level for various values of the Rotational Quantum Number $(l)$ as a function of $V_{0}$. We choose $=0.3$, and $\alpha=0.001$ in $3 D$. 


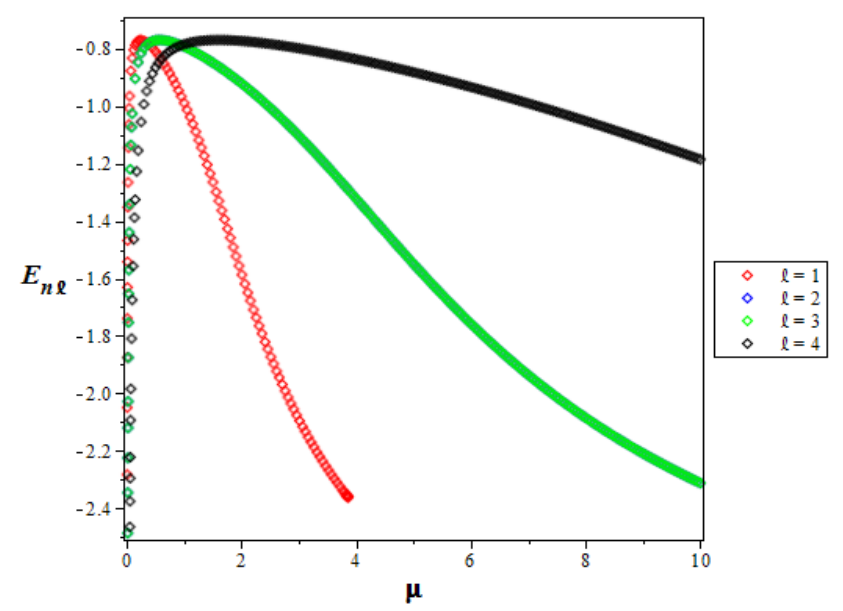

Fig. 11. The variation of the ground state $(n=0)$ energy level for various values of the Rotational Quantum $\operatorname{Number}(l)$ as a function of $\mu$. We choose $l=0.3, V_{0}=0.5$ and $\alpha=0.001$ in $3 D$.

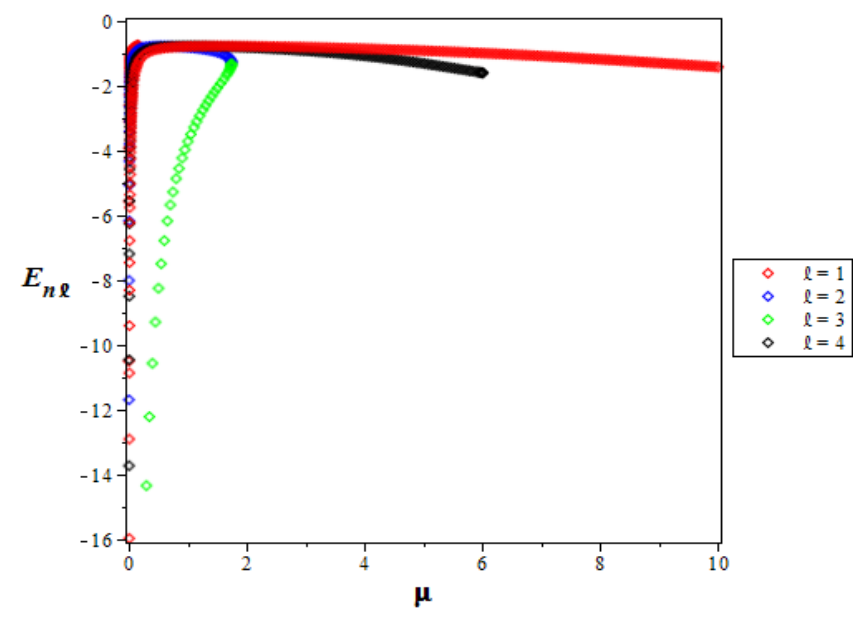

Fig. 12. The variation of the First excited state $(n=1)$ energy level for various values of the Rotational Quantum Number $(l)$ as a function of $\mu$. We choose $=0.3, V_{0}=0.5$ and $\alpha=0.001$ in $3 D$.

\section{DISCUSSION}

In our study, the energy eigenvalues of the Energy Dependent Generalized Inverse Quadratic Yukawa Potential (EDGIQYP) model were computed using (31), for different values of the energy slope parameters given $(\eta)$ which are presented in Table I-IV in 3D. When $\eta=0$, the energy equation (31) reduces to the Energy for Generalized Inverse Quadratic Yukawa Potential (EDGIQYP) model, and the corresponding numerical eigenvalues presented in Tables IIV for $\eta=0$ agrees perfectly with the result presented in Table 1 of [18] in the absence of the energy dependence.

We have plotted the shape of the Energy Dependent Generalized Inverse Quadratic Yukawa Potential (EDGIQYP) model in Fig. 1, 2. This figure gives an insight into the behaviour of the potential. Also, the variation of the energy eigenvalues with different parameters such as Dimensions $(D)$, coupling strength $V_{0}$ and particle mass $(\mu)$ are shown in Fig. 7-12 respectively, for various values of $n$ and $\ell$. In these figures, there is a decrease in energy eigenvalues as the potential strength increases (quasi asymptotic) in the ground state energy level. There was an increase in energy as dimensions' increases for both the the ground state and first excite energy levels respectively. It is evident also that as the energy slope parameter (for $\eta \geq 0$ ) increases the energy values increase too.

In Fig. 3 and 4, The variation of the ground state $(n=0)$ energy level and first excited state $(n=1$ state for various values of the energy slope $\operatorname{parameter}(\eta)$ as a function of $l$ were plotted. We choose $V_{0}=0.5$, and $\alpha=0.001$ in $3 D$. It was observed that as the rotational quantum number increases the energy increases for various values of $\eta$. In Fig. 5 and 6, the variation of the ground state $(n=0)$ and first excited state $(n=1$ states energy level for the different values of the energy slope parameter (for $\eta \leq 0$ ) as a function of $l$. We choose $V_{0}=0.5$, and $\alpha=0.001$ in $3 D$. In the first excited it is observed that the energy becomes more negative (more attractive) for increasing $\eta$ and $l$. In the ground state, there existed an irregular behaviour as some points broke off due to the presence complex values which are unacceptable for bound state solutions.

\section{CONCLUSION}

In this study, the approximate bound state solutions of the Schrodinger equation with Energy Dependent Generalized Inverse Quadratic Yukawa Potential (EDGIQYP) model were obtained, via the Nikiforov-Uvarov method. The energy eigenvalues of were computed and special cases considered. Our results were consistent with the results in available literature. The shape of the potential model was plotted, and this gives a better understanding to the behaviour of the potential model. The variation of the combined energy eigenvalue with the potential parameters $\left(D, V_{0}\right.$ and $\left.\mu\right)$ were also plotted. It was discovered that the energy eigenvalues decrease as the various potential strength $\left(V_{0}\right)$ increase in the ground state. The present study can be extended to scrutinize the thermodynamic properties of energy dependent systems [49]-[55]. More so, this study could be extended by applying this energy dependent models to study quarks [56]-[67].

\section{REFERENCES}

[1] Snyder, H., \& Weinberg, J. (1940). Stationary states of scalar and vector fields. Physical Review, 57(4), 307.; b) Schiff, L. I., Snyder, H., $\&$ Weinberg, J. (1940). On the existence of stationary states of the mesotron field. Physical Review, 57(4), 315.

[2] Boumali, A., Dilmi, S., Zare, S., \& Hassanabadi, H. (2017). Survey on density of states and saturation effect of spectrum for an energydependent harmonic interaction. Karbala International Journal of Modern Science, 3(4), 191-201.

http://dx.doi.org/10.1016/j.kijoms.2017.09.001.

[3] Abdelmalek Boumali and Malika Labidi, Shannon entropy and Fisher information of the one-dimensional Klein Gordon oscillator with energy-dependent potential, Modern Physics Letters A. Vol. 33, No. 6 (2018) 1850033 (26 pages). DOI: $10.1142 / \mathrm{S} 0217732318500335$

[4] Ikot, A. N., Hassanabadi, H., Maghsoodi, E., \& Zarrinkamer, S. (2013). Relativistic pseudospin and spin symmetries of the energy-dependent Yukawa potential including a Coulomb-like tensor interaction. Ukrainian journal of physics, (58, № 10), 915-924.

[5] Rizov, V. A., Sazdjian, H., \& Todorov, I. T. (1985). On the relativistic quantum mechanics of two interacting spinless particles. Annals of Physics, 165(1), 59-97.

[6] Todorov, I. T. (1971). Quasipotential equation corresponding to the relativistic Eikonal approximation. Physical Review D, 3(10), 2351.

[7] Lepage, G. P. (1977). Analytic bound-state solutions in a relativistic two-body formalism with applications in muonium and positronium. Physical Review A, 16(3), 863.

[8] Sazdjian, H. (1988). The scalar product in two-particle relativistic quantum mechanics. Journal of mathematical physics, 29(7), 16201633 . 
[9] Formanek, J., Lombard, R. J., \& Mareš, J. (2004). Wave equations with energy-dependent potentials. Czechoslovak journal of physics, 54(3), 289-315.

[10] Mustafa Salti, Oktay AYDOĞDU*, Spin (Pseudospin) doublet in view of energy-dependent potential.Turk J Phys (2017) 41: 112.doi:10.3906/_z-1602-14.

[11] H. Hassanabadi, S. Zarrinkamar and A. A. Rajabi, Commun. Theor. Phys. 55, 541 (2011).

[12] Abdelmalek Boumali, Samia Dilmi, Soroush Zare, Hassan Hassanabadi Survey on density of states and saturation effect of spectrum for an energy-dependent harmonic interaction (2017), http://dx.doi.org/10.1016/j.kijoms.2017.09.001.

[13] A.N. Ikot, H. Hassanabadi, E. Maghsoodi, and S. Zarrinkamar, DDimensional Dirac Equation for Energy-Dependent Pseudoharmonic and Mie-type Potentials via SUSYQM. Commun. Theor. Phys. 61 (2014) 436-446 Vol. 61, No. 4, April 1, 2014.

[14] A.N. Ikot, U.S. Okorie, A.T. Ngiangia, C.A. Onate, C.O. Edet, I.O. Akpan, P.O. Amadi, Bound state solutions of the Schrödinger equation with energy dependent molecular Kratzer potential via asymptotic iteration method, Eclet. Quím. J. 45 (2020) 65 , https://doi.org/10.26850/1678-4618eqj.v45.1.p65-76.

[15] Axel Schulze-Halberg and O" zlem Yes, iltas Generalized Schrödinger equations with energy-dependent potentials: Formalism and applications. Journal of Mathematical Physics 59, 113503 (2018).

[16] Hassanabadi, H., Maghsoodi, E., Oudi, R., Zarrinkamar, S., \& Rahimov, H. (2012). Exact solution Dirac equation for an energydependent potential. The European Physical Journal Plus, 127(10), 19.

[17] Hamzavi, M., \& Ikhdair, S. M. (2012). Relativistic study of the energydependent Coulomb potential including Coulomb-like tensor interaction. Canadian Journal of Physics, 90(7), 655-660.

[18] Oluwadare, O., \& Oyewumi, K. (2017). Non-relativistic treatment of a generalized inverse quadratic Yukawa potential. Chinese Physics Letters, 34(11), 110301.

[19] Falaye, B. J., Ikhdair, S. M., \& Hamzavi, M. (2015). Formula method for bound state problems. Few-Body Systems, 56(1), 63-78.

[20] C. O. Edet, P. O. Okoi, S. O. Chima, Analytic solutions of the Schrödinger equation with non-central generalized inverse quadratic Yukawa potential, Rev. Bras. Ens. Fís. 42 (2019) e20190083. https://doi.org/10.1590/1806-9126-RBEF-2019-0083.

[21] Hamzavi, M., Ikhdair, S. M., \& Ita, B. I. (2012). Approximate spin and pseudospin solutions to the Dirac equation for the inversely quadratic Yukawa potential and tensor interaction. Physica Scripta, 85(4), 045009 .

[22] Ikhdair, S. M. (2012). Approximate $\kappa$-state solutions to the DiracYukawa problem based on the spin and pseudospin symmetry. Central European Journal of Physics, 10(2), 361-381.

[23] Hassanabadi, S., Ghominejad, M., Zarrinkamar, S., \& Hassanabadi, H. (2013). The Yukawa potential in semirelativistic formulation via supersymmetry quantum mechanics. Chinese Physics B, 22(6), 060303.

[24] Edet, C. (2020). Effects of Magnetic And Aharanov-Bohm (AB) Fields on the Energy Spectra of the Yukawa Potential. arXiv preprint arXiv:2012.08644.

[25] U. S. Okorie, A. N. Ikot, C. O. Edet, I. O. Akpan, R. Sever and R. Rampho, Solutions of the Klein Gordon equation with generalized hyperbolic potential in D-dimensions, J. Phys. Commun. 3 (2019) 095015, https://doi.org/10.1088/2399-6528/ab42c6.

[26] [26] A.N. Ikot, U.S. Okorie, G. Osobonye, P.O. Amadi, C.O. Edet, M.J. Sithole, G.J. Rampho, R. Sever, Superstatistics of Schrödinger equation with pseudo-harmonic potential in external magnetic and Aharanov-Bohm fields, Heliyon 6 (2020) e03738, https://doi.org/10.1016/j.heliyon.2020.e03738.

[27] A. N. Ikot, U. S. Okorie, G. J. Rampho, P. O. Amadi, C. O. Edet, I. O. Akpan, H. Y. Abdullah \& R. Horchani, Klein-Gordon Equation and Nonrelativistic Thermodynamic Properties with Improved Screened Kratzer Potential. Journal of Low Temperature Physics, 1-21. (2021) https://doi.org/10.1007/s10909-020-02544-w.

[28] H. Louis, B.I. Ita, T.O. Magu, O.U. Akakuru, N.A. Nzeata-Ibe, A.I Ikeuba, A.I. Pigweh and C.O. Edet, Solutions to the Dirac Equation for Manning-Rosen Plus Shifted Deng-Fan Potential and Coulomb-Like Tensor Interaction Using Nikiforov-Uvarov Method, Intl. J. Chem. 10 (2018) 99, https://doi.org/10.5539/ijc.v 10 n 3 p 99.

[29] U S Okorie, C O Edet, A N Ikot, G J Rampho and R Sever, Thermodynamic functions for diatomic molecules with modified Kratzer plus screened Coulomb potential, Indian J Phys (2020) https://doi.org/10.1007/s12648-019-01670-w.

[30] C.O. Edet, K.O. Okorie, H. Louis and N.A. Nzeata-Ibe, Any l-state solutions of the Schrodinger equation interacting with Hellmann-
Kratzer potential model, Indian J Phys 94 (2020) 243 https://doi.org/10.1007/s12648-019-01477-9.

[31] C. O. Edet, P. O. Amadi, M. C. Onyeaju, U. S. Okorie, R. Sever, G. J Rampho, Hewa Y. Abdullah, Idris H. Salih and A. N. Ikot, Thermal Properties and Magnetic Susceptibility of Hellmann Potential in Aharonov-Bohm (AB) Flux and Magnetic Fields at Zero and Finite Temperatures. Journal of Low Temperature Physics (2020). https://doi.org/10.1007/s10909-020-02533-z.

[32] G. J. Rampho, A. N. Ikot, C. O. Edet \& U. S. Okorie, Energy spectra and thermal properties of diatomic molecules in the presence of magnetic and $A B$ fields with improved Kratzer potential, Mol. Phys, (2020) https://doi.org/10.1080/00268976.2020.1821922.

[33] B.I. Ita, H. Louis, O.U. Akakuru, N.A. Nzeata-Ibe, A.I. Ikeuba, T.O. Magu, P.I. Amos and C.O. Edet, Approximate Solution to the Schrödinger Equation with Manning-Rosen plus a Class of Yukawa Potential via WKBJ Approximation Method, Bulg. J. Phys. 45 (2018) 323. https://www.bjp-bg.com/paper1.php?id=914

[34] A. N. Ikot, C. O. Edet, P. O. Amadi, U. S. Okorie, G. J. Rampho, and H. Y. Abdullah, Thermodynamic properties of Aharanov-Bohm (AB) and magnetic fields with screened Kratzer potential, Eur. Phys. J. D 74 (2020) 159, https://doi.org/10.1140/epjd/e2020-10084-9.

[35] C O Edet, P O Okoi, A S Yusuf, P O Ushie and P O Amadi, Bound state solutions of the generalized shifted Hulthe'n potential, Indian J. Phys. (2019) https://doi.org/10.1007/s12648-019-01650-0.

[36] C.O. Edet, U.S. Okorie, A.T. Ngiangia and A.N. Ikot, Bound state solutions of the Schrodinger equation for the modified Kratzer potential plus screened Coulomb potential, Ind. J. Phys. 94 (2019) 425 , https://doi.org/10.1007/s12648-019-01467-x.

[37] H. Louis, B.I. Ita, O.U. Akakuru, N.A. Nzeata-Ibe, A.I. Ikeuba, T.O. Magu, P.I. Amos and C.O. Edet, $l$-state Solutions of the Relativistic and Non-Relativistic Wave Equations for Modified Hylleraas-Hulthen Potential Using the Nikiforov-Uvarov Quantum Formalism, Oriental J. Phys. Sci. 3 (2018) 1. http://www.orientjphysicalsciesnces.org/.

[38] P.O. Okoi, C.O. Edet and T.O.Magu, Relativistic treatment of the Hellmann-generalized Morse potential, Rev. Mex. Fis. 66 (2020) 1, https://doi.org/10.31349/RevMexFis.66.1.

[39] C.O. Edet and P.O. Okoi, Any 1-state solutions of the Schrödinger equation for q-deformed Hulthen plus generalized inverse quadratic Yukawa potential in arbitrary dimensions Rev. Mex. Fis. 65 (2019) 333, https://doi.org/10.31349/RevMexFis.65.333.

[40] Pekeris, C. L. (1934). The rotation-vibration coupling in diatomic molecules. Physical Review, 45(2), 98.

[41] Hamzavi, M., Ikhdair, S. M., \& Ita, B. I. (2012). Approximate spin and pseudospin solutions to the Dirac equation for the inversely quadratic Yukawa potential and tensor interaction. Physica Scripta, 85(4), 045009

[42] Onate, C. A., \& Ojonubah, J. O. (2016). Eigensolutions of the Schrödinger equation with a class of Yukawa potentials via supersymmetric approach. Journal of Theoretical and Applied Physics, $10(1), 21-26$

[43] C. A. Onate, Relativistic and Non-relativistic Solutions of the Inversely Quadratic Yukawa Potential, The African Review of Physics (2013) 8:0046 325

[44] Sameer M. Ikhdair Majid Hamzavi, Relativistic New Yukawa-Like Potential and Tensor Coupling Few-Body Syst (2012) 53:487-498, DOI 10.1007/s00601-012-0475-2.

[45] Ituen B. Okon, Oyebola Popoola, Cecilia N. Isonguyo and Akaninyene D. Antia, Physical Science International Journal 19(2): 1-27, 2018 Article no. PSIJ.43610 ISSN: 2348-0130.

[46] Isonguyo, C. N., Oyewumi, K. J., \& Oyun, O. S. (2018). Quantum information-theoretic measures for the static screened Coulomb potential. International Journal of Quantum Chemistry, 118(15), e25620.

[47] Dong, S. H., \& Sun, G. H. (2004). The Schrödinger equation with a Coulomb plus inverse-square potential in D dimensions. Physica Scripta, 70(2-3), 94.

[48] Oyewumi, K. J. (2005). Analytical solutions of the Kratzer-Fues potential in an arbitrary number of dimensions. Foundations of Physics Letters, 18(1), 75-84

[49] C.O. Edet, U.S. Okorie, G. Osobonye, A.N. Ikot, G.J. Rampho and R Sever, Thermal properties of Deng-Fan-Eckart potential model using Poisson summation approach, J. Math. Chem. 58 (2020) 989. https://doi.org/10.1007/s10910-020-01107-4.

[50] C.O. Edet, P.O. Amadi, U.S. Okorie, A. Tas, A.N. Ikot and G. Rampho, Solutions of Schrödinger equation and thermal properties of generalized trigonometric P"oschl-Teller potential. (2020). Rev. Mex. F1s. 66 (2020) 824. https://doi.org/10.31349/RevMexFis.66.824

[51] Ikot, A. N., Okorie, U. S., Amadi, P. O., Edet, C. O., Rampho, G. J., \& Sever, R. (2021). The Nikiforov-Uvarov-Functional Analysis (NUFA) Method: A New Approach for Solving Exponential-Type Potentials. 
Few-Body Systems, 62(1), 1-16. https://doi.org/10.1007/s00601-02101593-5.

[52] Edet, C. O., \& Ikot, A. N. (2021). Effects of Topological Defect on the Energy Spectra and Thermo-magnetic Properties of $\$$ \$ CO $\$$ \$ CO Diatomic Molecule. Journal of Low Temperature Physics, 203, 84-111 (2021). https://doi.org/10.1007/s10909-021-02577-9.

[53] Edet, C. O., Ikot, A. N., Onyeaju, M. C., Okorie, U. S., Rampho, G. J., Lekala, M. L., \& Kaya, S. (2021). Thermo-magnetic properties of the screened Kratzer potential with spatially varying mass under the influence of Aharanov-Bohm (AB) and position-dependent magnetic fields. Physica E: Low-dimensional Systems and Nanostructures, 131, 114710. https://doi.org/10.1016/j.physe.2021.114710.

[54] Ikot, A. N., Edet, C. O., Okorie, U. S., Abdel-Aty, A. H., Ramantswana, M., Rampho, G. J., ... \& Kaya, S. (2021). Solutions of the 2D Schrodinger equation and its thermal properties for Improved Ultra Generalized Exponential Hyperbolic potential (IUGE-HP). arXiv preprint arXiv:2104.02117.

[55] Edet, C., Ikot, A., Okorie, U., Abdullah, H., \& Salah, I. (2020). Eigenfunction, uncertainties and thermal properties of the Schrodinger equation with Screened modified Kratzer potential for diatomic molecules. Authorea Preprints. https ://doi.org/10.22541 /au.15909 8050.09308623 .

[56] Inyang, E. P., Inyang, E. P., Ntibi, J. E., Ibekwe, E. E., \& William, E. S. (2020). Approximate solutions of D-dimensional Klein-Gordon equation with Yukawa potential via Nikiforov-Uvarov method. Indian Journal of Physics, 1-7.

[57] Inyang, E. P., Inyang, E. P., William, E. S., \& Ibekwe, E. E. (2021). Study on the applicability of Varshni potential to predict the massspectra of the Quark-antiquark systems in a non-relativistic framework. arXiv preprint arXiv:2101.00333.

[58] Inyang, E. P., William, E. S., \& Obu, J. A. (2020). Eigensolutions of the N-dimensional Schrl" odinger equation interacting with VarshniHulthl'en potential model. arXiv preprint arXiv:2012.13826.

[59] Inyang, E. P., Inyang, E. P., William, E. S., Ibekwe, E. E., \& Akpan, I. O. (2020). Analytical Investigation of Meson Spectrum via Exact Quantization Rule Approach. arXiv preprint arXiv:2012.10639.

[60] William, E. S., Obu, J. A., Akpan, I. O., Thompson, E. A., \& Inyang, E. P. (2020). Analytical Investigation of the Single-Particle Energy Spectrum in Magic Nuclei of ${ }^{56} \mathrm{Ni}$ and ${ }^{116} \mathrm{Sn}$. European Journal of Applied Physics, 2(6).

[61] Inyang, E. P., Ntibi, J. E., Ibekwe, E. E., \& William, E. S. (2021) Analytical study on the Applicability of Ultra Generalized Exponential Hyperbolic Potential to Predict the Mass Spectra of the Heavy Mesons. arXiv preprint arXiv:2101.06389.

[62] Akpan, I. O., Inyang, E. P., \& William, E. S. (2021). Approximate solutions of the Schrodinger equation with Hulthen-Hellmann Potentials for a Quarkonium system. arXiv preprint arXiv:2101.01175.

[63] Inyang, E. P., Akpan, I. O., Ntibi, J. E., \& William, E. S. (2020). Analytical solutions of the Schrödinger equation with class of Yukawa potential for a Quarkonium system via series expansion method. European Journal of Applied Physics, 2(6).

[64] William, E. S., Inyang, E. P., \& Thompson, E. A. (2020). Arbitrary 1solutions of the Schrödinger equation interacting with HulthénHellmann potential model. Revista Mexicana de Fisica, 66(6 NovDec), 730-741.

[65] Ntibi, J. E., Inyang, E. P., Inyang, E. P., \& William, E. S. (2020). Relativistic Treatment of D-Dimensional Klien-Gordon equation with Yukawa potential. International Journal of Innovative Science, Engineering Technology, 11(7), 2348-7968.

[66] Inyang, E. P., Ntibi, J. E., WILLIAM, E., \& EKECHUKWU, C. (2020). Any State Solutions of the Schrödinger equation interacting with class Of Yukawa-Eckart potentials. Intl J. Innov Sci, Engr. Tech, 7(11).

[67] Ibekwe, E. E., Okorie, U. S., Emah, J. B., Inyang, E. P., \& Ekong, S. A. (2021). Mass spectrum of heavy quarkonium for screened Kratzer potential (SKP) using series expansion method. The European Physical Journal Plus, 136(1), 1-11. 